\title{
Síndrome de intestino irritable posterior a colecistectomía laparoscópica. Estudio de cohorte prospectivo
}

\author{
Irritable bowel syndrome posterior to laparoscopic \\ cholecystectomy. A prospective cohort study
}

\author{
Francisco Cabrera-Mendoza, ${ }^{*}$ Andrés García-Flores, ${ }^{\ddagger}$ Juan Ramírez-Cuesta, ${ }^{*}$ \\ Aurelio Barrera-González, ${ }^{*}$ Gregorio Villarreal-Treviño, ${ }^{*}$ Sergio Moya-González, \\ Anira Lizbeth Castro-Zárate, ${ }^{\ddagger}$ Sandra Gabriela Medina-Escobedo*
}

Palabras clave: Colecistectomía, laparoscopia, diarrea, síndrome de intestino irritable.

Keywords: Cholecystectomy, laparoscopy, diarrhea, irritable bowel syndrome.

* Departamento de Cirugía General. Hospital Regional Monterrey, ISSSTE, Monterrey, N.L. México. * Centro de Atención Médica, Universidad de Monterrey, Monterrey, N.L. México.

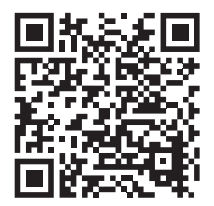

\section{RESUMEN}

Introducción: La colecistectomía laparoscópica es uno de los procedimientos quirúrgicos digestivos que se realiza con más frecuencia en el mundo. Se ha descrito que el síndrome de intestino irritable se presenta con mayor frecuencia en pacientes colecistectomizados, sobre todo el subtipo con predominio de diarrea. Objetivos: Evaluar la presencia de síndrome de intestino irritable antes y después de una colecistectomía laparoscópica. Material y métodos: Estudio de cohorte prospectivo, con grupo control. Se definió síndrome de intestino irritable de acuerdo con criterios de ROMA IV, se dio seguimiento al mes, tres, seis y 12 meses postoperatorios en busca de sintomatología. El análisis se realizó con prueba exacta de Fisher unidireccional. Resultados: De 166 pacientes, seis presentaron síndrome de intestino irritable más allá de los seis meses postoperatorios, el grupo control tuvo dos casos nuevos al término del seguimiento. Se presentaron ocho casos de síndrome postcolecistectomía. Conclusiones: No se puede establecer una relación diferente al azar entre pacientes intervenidos de colecistectomía laparoscópica y la presencia de síndrome de intestino irritable en cualquiera de sus variantes.

\section{ABSTRACT}

Introduction: Laparoscopic cholecystectomy is one of most performed surgical procedures around the world. Have been described an a posteriori association between irritable bowel syndrome and patients who underwent a laparoscopic cholecystectomy, mostly the diarrhea subtype. Objective: Evaluate the presence before and after a laparoscopic cholecystectomy. Material and methods: A prospective cohort study with control group. Irritable bowel syndrome was defined according to ROMA IV criteria, patients was evaluated at month one, three, six and 12 postoperatively looking for symptoms. An unidirectional fishers exact test was used for analysis. Results: For a total of 166 patients only six developed irritable bowel syndrome, diarrhea subtype; in the control group two patient developed irritable bowel syndrome during the follow-up. A total eight cases of postcholecystectomy syndrome were detected. Conclusions: A different than random association between cholecystectomized patients and the presence of irritable bowel syndrome in any of its variants cannot be established.

\section{Abreviaturas:}

ELV $=$ enfermedad litiásica vesicular

$\mathrm{CL}=$ colecistectomía laparoscópica

SII = síndrome de intestino irritable

SII-D = síndrome de intestino irritable subtipo diarrea

SII-C = síndrome de intestino irritable subtipo constipación

SII-M = síndrome de intestino irritable subtipo mixto

SPC $=$ síndrome postcolecistectomía

\section{INTRODUCCIÓN}

T a enfermedad litiásica vesicular (ELV) tiene $ـ$ alta prevalencia en la población occidental. ${ }^{1}$ La colecistectomía laparoscópica (CL) es uno de los procedimientos quirúrgicos digestivos que más se realiza en el ámbito mundial,

Citar como: Cabrera-Mendoza F, García-Flores A, Ramírez-Cuesta J, Barrera-González A, Villarreal-Treviño G, MoyaGonzález S et al. Síndrome de intestino irritable posterior a colecistectomía laparoscópica. Estudio de cohorte prospectivo. Cir Gen. 2021; 43 (1): 30-35. https://dx.doi.org/10.35366/103911 
en los Estados Unidos rebasa los 750,000 procedimientos por año. ${ }^{2}$ El síndrome de intestino irritable (SII) es un trastorno funcional digestivo común en la consulta general y en gastroenterología caracterizado por disconfort y dolor abdominal, anormalidad en hábitos intestinales, entre otros síntomas inespecíficos. ${ }^{3}$ Ryle notó que múltiples procedimientos quirúrgicos abdominales son notoriamente más comunes en pacientes con este síndrome, como histerectomías, apendicectomías y colecistectomías. ${ }^{4}$ El estudio colaborativo multicéntrico de Corazziari y colaboradores (MICOL) llegó a la conclusión que los pacientes con SII son más colecistectomizados que la población general. ${ }^{5}$ En un estudio restrospectivo del equipo de Amieva-Balmori, ${ }^{6}$ se evaluó la presencia de SIl y sus subtipos de acuerdo con los criterios de ROMA IV en un grupo de pacientes con antecedente de colecistectomía, encontrando que el síndrome de intestino irritable subtipo diarrea (SII-D) se presentó con mayor frecuencia en pacientes colecistectomizados. Existe dolor abdominal y molestias abdominales ${ }^{7}$ en una no despreciable cantidad de pacientes después de la colecistectomía, popularmente referidas; sin embargo, no descritas, entre las que destaca la diarrea; no obstante, no existen estudios prospectivos que demuestren esta asociación, motivo por el cual realizamos el presente estudio de cohorte prospectivo.

\section{MATERIAL Y MÉTODOS}

Estudio comparativo, prospectivo, de cohorte dinámica, controlado, realizado en el Hospital Regional de Monterrey del Instituto para la
Salud y Seguridad Social de los Trabajadores del Estado (ISSSTE) en casos intervenidos quirúrgicamente durante 2017 y seguidos durante 2018. Aprobado por el comité de ética e investigación de dicho hospital (folio 042, octubre de 2016). De acuerdo al muestreo probabilístico (diferencia de proporciones 10\%, ajustado a pérdidas de 5\%), se ingresaron 171 sujetos de estudio. No se informó a los pacientes sobre el objetivo de seguimiento, y no se les dieron recomendaciones dietéticas postoperatorias diferentes a las que habitualmente se dan para una dieta saludable, con el objetivo de disminuir el efecto Hawthorne. Se incluyeron aleatoriamente (simple, tabla de números) casos con diagnóstico ELV programados para CL y que no tuvieran datos de alarma gastrointestinal que sugirieran otros trastornos más graves; se excluyeron los pacientes con diagnóstico de neoplasias malignas digestivas, cirrosis hepáti$\mathrm{ca}$, hemorragia digestiva alta o baja, falla renal crónica, diabetes descontrolada o alteraciones psiquiátricas que impidieran el interrogatorio directo confiable, elegidos aleatoriamente de los ingresos al Servicio de Cirugía General de nuestro hospital. Se eliminaron para el análisis a los casos con SII previo a la cirugía a todos auqéllos que tuvieron conversión a cirugía abierta, que hubieran presentado alguna de las situaciones previamente descritas durante el transoperatorio, postoperatorio inmediato y seguimiento. El seguimiento en búsqueda de SII por criterios ROMA IV (Tabla 1) fue a los meses uno, tres, seis y 12 postoperatorios. El investigador que realizó la valoración inicial fue diferente al cirujano, así como al investigador del seguimiento posterior. Paralelamente, se

Tabla 1: Criterios diagnósticos ROMA IV.

\begin{tabular}{|c|c|c|c|}
\hline & \multicolumn{3}{|c|}{ Signos } \\
\hline $\begin{array}{c}\text { Al menos un día a la } \\
\text { semana (mínimo tres } \\
\text { meses): dolor abdominal* }\end{array}$ & $\begin{array}{c}\text { Relacionado a la } \\
\text { defecación }\end{array}$ & $\begin{array}{c}\text { Asociado a un cambio } \\
\text { de la frecuencia } \\
\text { de las heces }\end{array}$ & $\begin{array}{l}\text { Asociado a un cambio } \\
\text { en la forma de las heces } \\
\text { (escala de Bristol) }\end{array}$ \\
\hline
\end{tabular}

* La presencia de dolor abdominal sumado a uno o más de los signos mostrados es una condición necesaria para el diagnóstico.

Modificado de: Sebastián-Domingo JJ. ${ }^{8}$ 
Tabla 2: Distribución inicial de los grupos a seguir. Homogéneos por edad, sexo y prevalencia de síndrome de intestino irritable. $\mathrm{N}=336$.

\begin{tabular}{lccc} 
& $\begin{array}{c}\text { Pacientes programados } \\
\text { para colecistectomía } \\
\text { laparoscópica } \\
\mathbf{N}=\mathbf{~ 1 6 8}\end{array}$ & $\begin{array}{c}\text { Pacientes control } \\
\mathbf{N}=\mathbf{1 6 8}\end{array}$ & Homogeneidad \\
\hline Edad (media) años & 46.4 & $45.2 \pm 10.0$ & $\mathrm{p} \leq 0.01$ \\
Sexo & & & $\mathrm{p} \leq 0.01$ \\
$\quad$ Femenino & 105 & 105 & \\
$\quad$ Masculino & 63 & 63 & $\mathrm{p} \leq 0.01$ \\
SII (inicial) & 2 & 1 & \\
SII-D & 0 & 0 & \\
SII-C & 0 & 1 & \\
SII-M & 166 & 166 & \\
Sanos restantes & & & \\
\hline
\end{tabular}

SII = síndrome de intestino irritable, SII-D = síndrome de intestino irritable subtipo diarrea, SII-C = síndrome de intestino irritable subtipo constipación, SII-M = síndrome de intestino irritable subtipo mixto.

incluyó un grupo control pareado por edad y sexo, obtenido en forma aleatoria de la consulta médica general de dos de los investigadores, que fue seguido presencial y telefónicamente en los mismos periodos de tiempo, en búsqueda de síntomas compatibles para SII de acuerdo con ROMA IV; esto para ambos grupos en los periodos mencionados. ${ }^{8}$ Los datos fueron recolectados en hoja de datos electrónica, de la historia clínica del paciente que incluyeron todos los cuestionarios tanto preoperatoriamente como al seguimiento. Se tabularon los datos obtenidos para calcular el riesgo relativo, aumento absoluto de riesgo, y prueba exacta de Fisher unidireccional, tomando como significativo un valor de p menor a 0.05 .

\section{RESULTADOS}

De un total de 171 casos programados con ELV durante el periodo mencionado, se eliminaron dos por pérdida de seguimiento, y uno más ante el hallazgo de cáncer de vesícula biliar; y para el análisis estadístico se eliminaron dos con SII previo a la CL (pérdidas totales: cinco casos, 2.92\%). Del total de pacientes (166) intervenidos quirúrgicamente, 91.57\% (152) fue de manera electiva; $8.43 \%$ (14) se ingresaron por medio de urgencias para una CL temprana por colecistitis aguda, con media de tiempo operatorio de $92 \pm 51$ minutos y porcentaje de conversión de cero (Tabla 2). A la valoración inicial, se encontraron dos casos de SII subtipo diarrea en el grupo de casos que se iban a someter a $\mathrm{CL}$; se presentaron durante el seguimiento seis más de SII subtipo diarrea en el grupo intervenido quirúrgicamente, con síntomas persistentes incluso a los 12 meses del postoperatorio (SII-D propiamente dicho) (Tabla 3). Se destaca que ocho pacientes presentaron síntomas (entre ellos diarrea) desde los primeros días postoperatorios compatible con síndrome postcolecistectomía (SPC), los cuales remitieron dentro de las primeras cuatro semanas postoperatorias, y fueron pacientes diferentes a los que presentaron SII. En el grupo control, inicialmente se encontraron dos casos, uno subtipo diarrea y otro subtipo mixto; y durante el periodo de seguimiento, se presentaron dos casos nuevos, igualmente distribuidos. El análisis estadístico mostró un riesgo relativo de 3.00 (0.61-14.54), el aumento de riesgo absoluto (ARA o ARI, Absolute Risk Increase) es de 2.41 para SII-D, con valor de $p=0.0875$ (no significativo estadísticamente). Tomando en cuenta los pacientes que presentaron diarrea, 
dentro del espectro de síntomas del SPC, en comparación con la población abierta, tienen un riesgo relativo (RR) de 4 (0.86-18.55), aumento de riesgo absoluto de 4.05, con valor de $p=0.032$ (significativo), y 28.5 como número necesario para daño. Si bien el RR parece ser alto, no es significativo, y esa asociación parece estar dada por el azar (otras variables). Para el síndrome postcolecistectomía, el RR es cuatro veces mayor; el número necesario para daño nos hace concluir que hasta uno de cada 28 pacientes sometidos a $\mathrm{CL}$ puede desarrollar este síndrome, el cual es transitorio, y un limitado número de pacientes desarrollará diarrea crónica, aparentemente sin relación directa y completa con la falta de vesícula biliar. Existen otras variables que deben ser estudiadas.

\section{DISCUSIÓN}

La evidencia ${ }^{5,6}$ derivada de estudios retrospectivos sugiere que los pacientes que desarrollan
SII, principalmente el subtipo diarrea (SII-D), tienen antecedentes de colecistectomía, ${ }^{9}$ sin diferencia entre convencional o laparoscópica. En la literatura quirúrgica clásica, ${ }^{10,11}$ se ha aludido a que existe diarrea derivada del procedimiento quirúrgico como una posible complicación, incluso crónica, y de allí el que surgieran las recomendaciones de la restricción de alimentos grasos en la dieta posterior al procedimiento quirúrgico, información que ha transcurrido de generación en generación en la enseñanza quirúrgica, aunque sin evidencia sustancial. Consideramos que no existe evidencia sólida que sustente esta práctica, como lo demuestra este trabajo. Un estudio epidemiológico reportó mayor frecuencia de SII en pacientes operados de histerectomía y colecistectomía; ${ }^{12}$ y otro estudio realizado en la clínica Mayo concluye que los colecistectomizados tienen 2.2 mayor riesgo de presentar síndrome de intestino irritable. ${ }^{13}$ Manríquez y colaboradores, en un estudio de 100 pacientes (muestra no probabilística), 15\%

\section{Tabla 3: Casos nuevos de síndrome de intestino irritable presentados al seguimiento. $\mathrm{N}=166$.}

\begin{tabular}{|c|c|c|c|c|}
\hline & \multicolumn{4}{|c|}{ Meses } \\
\hline & 1 & 3 & 6 & 12 \\
\hline \multicolumn{5}{|c|}{ Pacientes sometidos a colecistectomía laparoscópica } \\
\hline Síndrome postcolecistectomía & $8^{*}$ & 0 & 0 & 0 \\
\hline SII-D & 0 & 6 & 6 & $6^{\ddagger}$ \\
\hline SII-C & 0 & 0 & 0 & 0 \\
\hline \multirow[t]{2}{*}{ SII-M } & 0 & 0 & 0 & 0 \\
\hline & \multicolumn{3}{|c|}{ Sanos restantes $=160$} & Total $=6$ \\
\hline \multicolumn{5}{|l|}{ Pacientes control } \\
\hline SII-D & 0 & 0 & 1 & $1^{\ddagger}$ \\
\hline SII-C & 0 & 0 & 0 & 0 \\
\hline SII-M & 0 & 1 & 1 & $1^{\ddagger}$ \\
\hline Total & \multicolumn{3}{|c|}{ Sanos restantes $=164$} & Total $=2$ \\
\hline
\end{tabular}

* Para síndrome postcolecistectomía, se realiza un análisis por separado, los casos de este síndrome se presentaron de manera rápida y se autolimitaron con la misma rapidez.

‡ Los pacientes que presentaron síndrome de intestino irritable fueron diferentes, a los que presentaron síndrome postcolecistectomía. No se incluyeron en el análisis los pacientes con diagnóstico previo de síndrome de intestino irritable. Para fines descriptivos, continuaron exactamente con los mismos síntomas después de la colecistectomía laparoscópica.

SII = síndrome de intestino irritable, SII-D = síndrome de intestino irritable subtipo diarrea, SII-C = síndrome de intestino irritable subtipo constipación, SII-M = síndrome de intestino irritable subtipo mixto. 
presentaron diarrea postoperatoria y en $8 \%$ esta condición la tuvieron de manera crónica, en el resto remitió dentro de los primeros 28 días; ${ }^{14}$ nosotros obtuvimos datos similares en nuestro grupo, pero con una muestra probabilística. La teoría que sustentan las conclusiones actuales es resultado de algunos experimentos en los que se evaluó la retención de algunos ácidos biliares radiomarcados en pacientes con SII, los cuales correlacionan con la severidad de síntomas, sobre todo en las variedades mixta alternante y predominio de diarrea. ${ }^{15}$ También ha sido descrito el síndrome postcolecistectomía, ${ }^{7}$ el cual debe ser diferenciado del SII-D por predilección matutina de los síntomas, intolerancia a los alimentos grasos, náusea, vómito, aerogastria, aerocolia y, en algunos casos, urgencia fecal ${ }^{16}$ que claramente se alivia al evacuar. La fisiopatología de este síndrome está excelentemente descrita por Jaramillo y Otero, ${ }^{17}$ la malabsorción de ácidos biliares o el incremento en su aporte aumentan las concentraciones de éstos en el colon, lo que modifica el desplazamiento de agua y electrólitos, ocasionando diarrea osmótica; la cual podría deberse a una causa crónica poco diagnosticada, sobre todo en casos colecistectomizados ${ }^{18}$ y su posible tratamiento con secuestradores de ácidos biliares. ${ }^{19}$ Sin embargo, la evidencia proveniente de estudios clínicos no está diseñada para demostrar causalidad; 6,7,20,21 por lo tanto, deben existir otras variables, además de los ácidos biliares en la fisiopatología de la diarrea crónica. Las revisiones sobre SII siguen colocando al antecedente de colecistectomía en significativa relación estadística con SII por criterios de ROMA. ${ }^{22-24}$

\section{CONCLUSIONES}

Nuestro equipo concluye que no hay una asociación diferente al azar entre una colecistectomía laparoscópica y el desarrollo de síntomas de dolor abdominal y diarrea crónica intermitente compatible con el diagnóstico de SII-D.

\section{REFERENCIAS}

1. Reynolds W. The first laparoscopic cholecystectomy. JSLS. 2001; 5: 89-94.

2. Keus F, Gooszen HG, van Laarhoven CJ. Open, smallincision, or laparoscopic cholecystectomy for patients with symptomatic cholecystolithiasis. An overview of Cochrane Hepato-Biliary Group reviews. Cochrane Database Syst Rev. 2010; 2010: CD008318.

3. Longstreth GF, Yao JF. Irritable bowel syndrome and surgery: a multivariable analysis. Gastroenterology. 2004; 126: 1665-1673.

4. Owens DM, Nelson DK, Talley NJ. The irritable bowel syndrome: long-term prognosis and the physicianpatient interaction. Ann Intern Med. 1995; 122: 107-112.

5. Corazziari E, Attili AF, Angeletti C, De Santis A. Gallstones, cholecystectomy and irritable bowel syndrome, MICOL population-based study. Dig Liver Dis. 2008; 40: 944-950.

6. Amieva-Balmori M, Azamar-Jacome AA, Rojas-Carrera SI, Cano-Contreras AD, Remes Troche JM. Prevalencia del síndrome de intestino irritable en pacientes con antecedentes de colecistectomía. ¿Existe alguna asociación? Med Int Méx. 2016; 32: 161-168.

7. Ros E, Zambon D. Postcholecystectomy symptoms. A prospective study of gallstone patients before and two years after surgery. Gut. 1987; 28: 1500-1504.

8. Sebastián-Domingo JJ. Los nuevos criterios de ROMA (IV) de los trastornos funcionales digestivos en la práctica clínica. Med Clin (Barc). 2017; 148: 464-468.

9. Rey E, Talley NJ. Irritable bowel syndrome: novel views on the epidemiology and potential risk factors. Dig Liver Dis. 2009; 41: 772-780.

10. Stinton LM, Myers RP, Shaffer EA. Epidemiology of Gallstones. Gastroenterol Clin North Am. 2010; 39 (2): 157-169.

11. Dooley JS, Gurusamy KS, Davidson BR. Gallstones and benign biliary disease. En: Sherlock S. Diseases of the liver and biliary system. 13va ed. Oxford: Blackwell Science; 2018, 256-274.

12. Heaton KW, Parker D, Cripps H. Bowel function and irritable bowel symptoms after hysterectomy and cholecystectomy-a population based study. Gut. 1993; 34: 1108-1111.

13. McNally MA, Locke GR, Zinsmeister AR, Schleck $\mathrm{CD}$, Peterson J, Talley NJ. Biliary events and an increased risk of new onset irritable bowel syndrome: a population-based cohort study. Aliment Pharmacol Ther. 2008; 28: 334-343.

14. Manríquez E, Tejos R, Rojas A, Pimentel E, Vega T, Achurra $\mathrm{P}$, et al. Postcholecystectomy diarrhea is a frequent problem? Chil Cir. 2017; 69: 376-381.

15. Bajor A, Tornblom H, Rudling M, Ung KA, Simren M. Increased colonic bile acid exposure: a relevant factor for symptoms and treatment in IBS. Gut. 2015; 64: 84-92.

16. Espinosa AD, Espinosa RAA. Síndrome postcolecistectomía: problema frecuente, poco tratado. Rev Cub Med. 2014; 53: 337-347.

17. Jaramillo SR, Yurgaky SJ, Otero RW. Diarrea postcolecistectomía, enfoque diagnóstico y terapéutico. Revista Med. 2017; 25: 96-104.

18. Bielsa-Fernández MV. Diarrea y absorción deficiente. Rev Gastroenterol Mex. 2012; 77: 35-36.

19. Camilleri M, Acosta A, Busciglio A, Boldingh R, Dyer $R B$, Zinsmeister AR, et al. Effect of colesevelam on faecal bile acids and bowel functions in diarrhoeapredominant irritable bowel syndrome. Aliment Pharmacol Ther. 2015; 41: 438-448. 
20. Fisher M, Spillias DC, Tong LK. Diarrhoea after laparoscopic cholecystectomy: incidence and main determinants. ANZ J Surg. 2008; 78: 482-486.

21. Tuan-Pin Y, Fong-Ying C, Tsyr-En L, Mao-Te C. Diarrhea after laparoscopic cholecystectomy: associated factors and predictors. Asian J Surg. 2014; 37: 171-177.

22. Aziz I, Mumtaz S, Bholah H, Chowdhury UF, Sanders SD, Ford CA. High prevalence of idiopathic bile acid diarrhea among patients with diarrheapredominant irritable bowel syndrome based on rome III criteria. Clin Gastroenterol Hepatol. 2015; 13: 1650-1655.e2.

23. Madrid-Silva AM, Defilippi-Caffri C, Landskron-Ramos G, Olguín-Herrera F, Reyes-Ponce A, Castro-Lara A, Larraín-Corp S, Martínez-Roje N, Cortés-Espinoza J. The prevalence of irritable bowel symptoms in a population of shopping mall visitors in Santiago de Chile. Rev Gastroenterol Mex. 2013; 78: 203-210.

24. León-Barúa R. Chronic diarrhea post cholecistectomy. Rev Gatroenterol Peru. 2013; 33: 82-84.

Consideraciones y responsabilidad ética: Los procedimientos en humanos deben ajustarse a los principios establecidos en la Declaración de Helsinki de la Asociación Médica Mundial (AMM) y con lo establecido en la Ley General de Salud Título Quinto y Reglamento de la Ley General de Salud en Materia de Investigación para la Salud, y NOM-012SSA3-2012, que establece los criterios para la ejecución de proyectos de investigación para la salud en seres humanos, así como con las normas del Comité de Ética en Investigación de la institución donde se efectúen. En caso de tener número de registro proporcionarlo.

Financiamiento: Recursos propios de los autores. Conflicto de intereses: Sin conflicto de intereses.

Correspondencia:

Francisco Cabrera-Mendoza

E-mail: cabrera_md@icloud.com 